\title{
Moving Objects Detection by Conflict Analysis in Evidential Grids
}

\author{
Julien Moras, Véronique Cherfaoui, Philippe Bonnifait
}

\begin{abstract}
Advanced Driving Assistance Systems exploit exteroceptive sensors to help the driver in perceiving the dynamic environment, like other vehicles or pedestrians. This paper proposes an original approach to deal with this perception challenge in urban environments. The method detects mobile objects motions using grids elaborated thanks to a lidar range scanner and an enhanced map of the drivable space. The data fusion is performed using the Dempster-Shafer theory which provides an interesting framework particularly well adapted to manage the uncertainties of the sensors. By analyzing conflicting information, objects movements can be efficiently characterized. This formalism provides also the interesting possibility to introduce decay factors that are useful for forgetting old information. Experimental results obtained with an IBEO Alasca and an Applanix positioning system show that such a perception strategy can be effective compared to deterministic accumulation strategies.
\end{abstract}

\section{INTRODUCTION}

Nowadays, Advanced Driving Assistance Systems (ADAS) are promising technologies which will appear on many vehicles, like Electronic Stability Programs or Speed Regulators that are commonly used in curren vehicles. This next generation of ADAS is coming out and will be the basis of auto-braking systems or overtaking assistance systems. In such kind of application, reliable perception of the environment is crucial to avoid collisions and eliminate false alarms while reducing missed detections. An essential part of the perception problem is the detection of mobile objects because they constitute the more difficult part of the problem when using lidar technology. The velocity of the objects hit by the laser beam is not measured in opposition to a radar exploiting the Doppler effect.

Mobile Object Tracking (MOT) has been studied for a long time and many approaches have been proposed [1]. Classical MOT systems are based on features and rely on two principal steps: the clustering of the sensor raw data for object detection and a simultaneous tracking stage. The clustering step can be done for instance by image processing [2] or by clustering a set of lidar scan points [3]. This process provides a set of features referenced in the sensor frame. Single Hypothesis Tracking (SHT) systems [4] associate the detected objects with the tracks previously registered, using a specific strategy like JPDA (Joint Probabilistic Data Association, see e.g. [5]), or create new tracks. The update of the tracks is then done with a filtering stage based on the Kalman Filter. Multi-hypothesis tracking systems keep in memory several hypotheses which are updated using the detected features while unlikely hypotheses are rejected.

All authors are with Heudiasyc,CNRS UMR 6599,

Université de Technologie de Compiègne, 60200 Compiègne, France Contact author email: julien.moras@hds.utc.fr
Feature-based approaches are some times coupled with grid approaches in order to manage the mobile objects in complement to the grid framework that is dedicated to the static part [6]. Indeed, occupancy grids have been used for mobile robotics in static environments. New approaches have appeared in the last years, like the Bayesian Occupancy Filter (BOF) [7] which is able to estimate what is evolving in the grid. This approach uses a grid built in the sensor frame and estimates simultaneously the occupancy and the relative speed of each cell. This is performed trough the use of a probabilist association of the cells along the time.

In this paper, we focus on the detection of moving obstacles using only a lidar sensor. This kind of range sensor provides two types of information: the presence of echoes in the different scanned lines-of-sight, and their relative positions. The problem we are addressing is therefore to infer the dynamics of the scene by using lidar echoes. In this study, we consider the case of an equipped vehicle which evolves on a planar surface, like a road. We suppose that the pose of the vehicle is precisely provided by an external localization system. In the experiments, we used an Applanix system. The main contribution of this paper is to present a new way to detect mobile objects in the surrounding of the vehicle using a Belief Function (also called Evidential in this paper) framework coupled with a grid representation. The main benefit of this approach is that there is no assumption on the shape of the objects which is suitable for a wide range of urban obstacles like pedestrians, vehicles, or bicycles. In this strategy there is no feature detection and association step. Moreover, decay factors are introduced to model the fact that perception memory fades due to the passage of time. Finally, we present an interesting approach to determine the direction of mobile objects.

The paper is organized as follows. In the first section, we present the evidential occupancy grid framework that is used in the data fusion process. Then, a lidar model is proposed to obtained evidential measurements from the raw sensor data. In section III-A, the fusion processing core is detailed. We present how the detection of the mobile objects is performed and how a decay strategy is applied on the perceived grid. Finally, real experimental results are given and compared to a classical accumulation strategy.

\section{EVIDENTIAL GRIDS}

\section{A. Evidential grid framework}

An occupancy grid is a discrete representation of the environment which splits the space into a set of cells that may be free or occupied. Usual approaches attempt to determine the probability that some cell is occupied from 
sensor data. The grid framework was proposed by Elfes [8], but it remained little used at that time because this approach is time, memory and computation consuming. During the recent years, this representation has become more and more popular because it is an efficient framework for vehicle navigation and because embedded computers have more powerful calculation capabilities (memory, multi-core, GPU, etc...). Bayesian inference is the common background used to cope with errors and uncertainty and many extensions have been published in the literature [7], [9].

In this work, we propose to use an evidential approach based on Dempster-Shafer (DS) theory because this framework allows making the difference between unknown (there is no information) and doubt caused by conflicting information gathered incrementally in the fusion process. Similar ideas have been developed in [10], [11] to implement evidential occupancy grids intended to manage uncertainty arising from range sensors in the representation of the environment.

Let us study now how these concepts are handled. In a way similar to probabilistic grids, the frame of discernment includes two possible states: $\operatorname{Free}(F)$ and $\operatorname{Occupied}(O)$. In DS theory, this set is augmented by considering the power-set which is defined as $2^{\Omega}=\{\emptyset,\{F\},\{O\},\{F, O\}\}$, where $\Omega=\{F, O\}$ represents the ignorance in the same way as in [11]. For each cell, a mass function $m$ is calculated and provides a quadruplet on the state of the cell $\left[\begin{array}{llll}m(F) & m(O) & m(\Omega) & m(\emptyset)\end{array}\right]^{\prime}$. For an element $A$, $m(A)$ can represent the piece of evidence that the space is free $(A=F)$, occupied $(A=O)$ or unknown $(A=\Omega)$. The piece of evidence on the empty set, $m(\emptyset)$, is generally null, unless it results of a conflicting combination. $m$ verifies property: $\sum_{A \subseteq \Omega} m(A)=1$.

The Transferable Belief Model (TBM) framework proposed by Smets [12] provides also powerful tools (plausibility, belief, mass, pignistic probability) and many fusion operators have been developed in order to cope with different kinds of problems.

The strategy used in this paper manages two grids: an instantaneous grid computed from one scan called ScanGrid (SG) that will be fused into a global grid called MapGrid (MG).

\section{B. Sensor Model}

In this section, we propose a SG sensor model that attributes an initial mass $m_{S}$ (denoted in the following "BBA", for "Basic Belief Assignment") for each cell, depending on the reliability of the sensor. The SG captures the information coming from one lidar scan at time $k$.

Sensors provide data elaborated from physical phenomenas. To interpret such an information, one has to model the measurement method by making use of some simplifying assumptions. This paper focusses on lidar sensors and we propose here an evidential model for them. At every time step, a lidar provides a scan which is a set of laser telemetric points measured during one rotation. Even if the scanning process takes time [13], we consider it as instantaneous, e.g. all points have the same date.
Let us assume that the different laser beams are above the road i.e. the echoes are coming from potential obstacles. In order to be as close as possible to the sensor acquisition process, we make a $2 \mathrm{D}$ polar grid interpretation of the data. The space is divided in several angular sectors, each sector being divided in several cells along the range and each cell containing a mass function computed from the sensor model. The SG can be represented by four classical grids, using one for the mass of each state. Figure 1 illustrates each component of the grid for one echo.

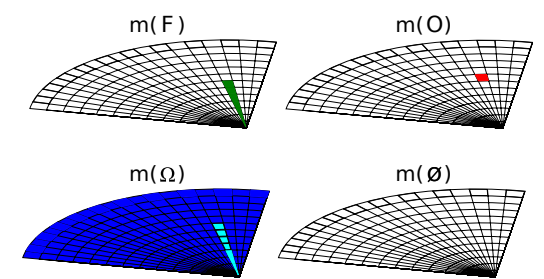

Fig. 1: Polar evidential representation, colors intensity refers to the value of the mass

Since a lidar scan captures a sparse representation of the real world, one has to compute the probability for an object to be hit by the laser beam in one scan. Let $\lambda_{M D}$ and $\lambda_{F A}$ be two coefficients which correspond respectively to the probability of missed detection and the probability of false alarm. $\lambda_{F A}$ depends essentially on sensor noise. $\lambda_{M D}$ is mainly related to the reflexivity of the target and to the blind area between two consecutive laser beams, when the beam divergence $\varphi$ is smaller than the angular step width $\beta$ (see Fig. 2). Since the reflective and geometrical properties of the objects in the scene are often unknown, we have developed a model that is only based on the geometrical coverage of the laser scans.

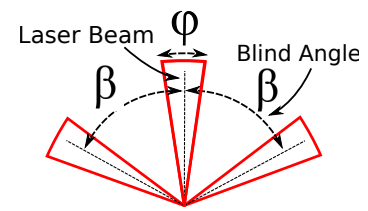

Fig. 2: Laser scanning coverage and blind angles

Because of the angular resolution of the map and the multi echoes and multi layers new lidar capabilities (multi echoes and multi layers), several points being in different cells can be located in the same angular sector, as illustrated by Figure 3. Classical lidar grid approaches doesn't handle this kind of configuration [14].

Let suppose that the angular precision of the lidar is very good compared to the grid angular resolution. Under this assumption, the sectors of the SG can be evaluated independently. Basically, if the laser beam returns an echo in a cell in one angular sector, then this cell is occupied, the cells crossed before are free and the cells behind are unknown. Now, if there are several laser beams in the same angular sector, they are handled together for quantifying the BBA of the SG sector (see Fig. 3). 


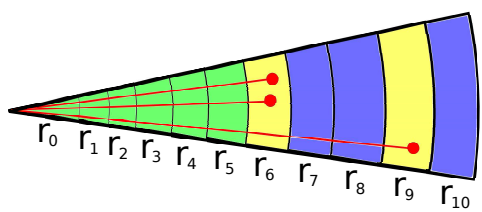

Fig. 3: Example of an angular sector of the SG with three lidar points ; colors refers to the most likely state of the cell (green $F$, yellow $O$, blue $\Omega$ )

In each row of the $\mathrm{SG}$ corresponds to an angular sector denoted by $\Theta=\left[\theta^{-} \theta^{+}\right]$. One cell is a box $R \times \Theta$, where $R=\left[r^{-} r^{+}\right]$refers to the range of the cell. Let denote $m_{S, k}\{\Theta, R\}(A)$ the piece of evidence attached to $A$, for the cell $R \times \Theta$, for the grid $S$ ( $S$ stands for ScanGrid).

Let $Z_{\Theta}$ be the set of $n$ scan points in the angular sector $\Theta:$

$$
Z_{\Theta}=\left\{z_{i}=\left[r_{i} \theta_{i}\right] \mid \theta_{i} \in \Theta, i \in[0, n]\right\}
$$

For every column $R$ of the row $\Theta$, the mass function quadruplet $\left[m_{S}(O) m_{S}(F) m_{S}(\Omega) m_{S}(\emptyset)\right]^{\prime}$ is given by $\forall i \in[0, n]$ :

$$
\begin{aligned}
& m_{S}\{\Theta, R\}(O)= \begin{cases}1-\lambda_{F A} & \text { if } \exists r_{i} \in R \\
0 & \text { otherwise }\end{cases} \\
& m_{S}\{\Theta, R\}(F)= \begin{cases}1-\lambda_{M D} & \text { if } r^{+}<\min \left(r_{i}\right) \\
0 & \text { otherwise }\end{cases} \\
& m_{S}\{\Theta, R\}(\Omega)= \begin{cases}\lambda_{F A} & \text { if } \exists r_{i} \in R \\
\lambda_{M D} & \text { if } r^{+}<\min \left(r_{i}\right) \\
1 & \text { otherwise }\end{cases} \\
& m_{S}\{\Theta, R\}(\emptyset)=0
\end{aligned}
$$

\section{Mobile ObJeCtS DETECTION BY GRID FUSION}

\section{A. Fusion process}

Several successive scans are needed to create a map in order to detect mobile objects in the scene [15]. In this part we detail how a grid in a global world frame (MapGrid) is built to map the navigable space and the environment (static and mobile). The MG is a Cartesian map referenced in a world frame and composed of $L$-length squared cells. Each cell refers to an occupancy mass function defined on $2^{\Omega}$ as described in part II-A. $m_{M}\{i, j\}()$ is the mass function assigned to the cell $(i, j)$ of $\mathrm{MG}(k)$. The MapGrid can be initialized using no prior information, i.e. with vacuous mass $m_{\Omega}$ on every cell $(m(\Omega)=1)$. As the fusion is done cell by cell in the full grid, we omit the indices of the cells of grid. So, $m_{M}$ refers to $\forall i, j, m_{M}\{i, j\}$.

The updating mechanism is sequential. At a given time sample, the previous MapGrid, $\mathrm{MG}(k-1)$ is updated with the current ScanGrid SG(k) built from the sensor measurements. The result is a new MapGrid MG(k). The updating step allows the classification of the cells containing moving objects. The proposed scheme is composed of five phases as described in Figure 4.

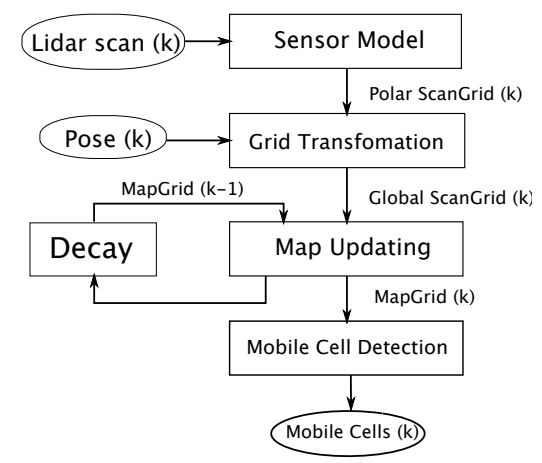

Fig. 4: Fusion architecture

Grid transformation: To be able to merge the information contained in the SG, we have to transform the grid to be spatially compatible with the MapGrid. This is achieved in two stages. First, the SG is projected from polar to Cartesian coordinates which induces approximations for cells that are far away from the vehicle due to size of the polar cell that increases with respect to the range. Then, the SG is moved from the sensor frame into the reference frame of the MapGrid. This requires the knowledge of the absolute pose of the vehicle at time $k$. Both transformations are achieved by using bi-linear interpolation methods on each piece of evidence of mass functions. The resulting grid $S^{\prime}$ is called Global ScanGrid (GSG) and each cell contains a new mass function $m_{S^{\prime}}$ (see [16] for details).

Decay factors: In dynamic environment, information becomes aging as time passes. Since no grid evolution model is considered, we cannot predict the MG. To handle this, we propose to determine a decay factor $\alpha$ resulting from the time difference $\Delta t=t_{S}-t_{M}$ and the time constant $\tau \cdot t_{M}$ is the time of the last update of the $\mathrm{MG}, t_{S}$ refers to the time of the current SG. $\tau$ represents the validity time of the cell information. The decay factor is an exponential decay function as described by $\alpha=e^{-\frac{\Delta t}{\tau}}$. This aging process consists in computing ${ }^{\alpha} m_{M}=$ decay $\left(m_{M}\right)$ with equation 2:

$$
\begin{cases}{ }^{\alpha} m_{M}(A)=\alpha \cdot m_{M}(A) & A \subset \Omega \\ { }^{\alpha} m_{M}(\Omega)=1-\alpha+\alpha \cdot m_{M}(\Omega) & \end{cases}
$$

Fusion: When a new Global ScanGrid $\operatorname{GSG}(k)$ is computed, it is fused with the previous MapGrid MG( $k-1)$ by the mean of the Dempster's rule (Eq. 3). Each cell is considered independent from the others ones. Fusion is performed using first the conjunctive combination rule and then the Dempster normalization. The conjunctive rule is detailed in Eq. 4. Before the Dempster's normalization (Eq. 5 ), we store the terms of the empty set mass $m_{\cap M, t}(\emptyset)$. This step is crucial for the mobile cells detection process.

$$
m_{M, k}={ }^{\alpha} m_{M, k-1} \oplus m_{S^{\prime}, k}
$$




$$
\begin{aligned}
& m_{\cap M, k}(O)=m_{S^{\prime}, k}(O) \cdot{ }^{\alpha} m_{M, k-1}(O)+m_{S^{\prime}, k}(\Omega) \cdot{ }^{\alpha} m_{M, k-1}(O) \\
& +m_{S^{\prime}, k}(O) \cdot{ }^{\alpha} m_{M, k-1}(\Omega) \\
& m_{\cap M, k}(F)=m_{S^{\prime}, k}(F) \cdot{ }^{\alpha} m_{M, k-1}(F)+m_{S^{\prime}, k}(\Omega) \cdot{ }^{\alpha} m_{M, k-1}(F) \\
& +m_{S^{\prime}, k}(F) \cdot{ }^{\alpha} m_{M, k-1}(\Omega) \\
& m_{\cap M, k}(\Omega)=m_{S^{\prime}, k}(\Omega) \cdot{ }^{\alpha} m_{M, k-1}(\Omega) \\
& m_{\cap M, k}(\emptyset)=m_{S^{\prime}, k}(O) \cdot{ }^{\alpha} m_{M, k-1}(F)+m_{S^{\prime}, k}(F) \cdot{ }^{\alpha} m_{M, k-1}(O) \\
& \begin{array}{lcc}
m_{M, k}(A)= & \frac{m_{\cap M, k}(A)}{1-m_{\cap M, k}(\emptyset)} & A \neq \emptyset \\
m_{M, k}(\emptyset)= & 0 & \text { else }
\end{array}
\end{aligned}
$$

B. Mobile Object detection and Motion Detection by analyzing the empty mass

Let us focus on the behavior of the Dempster combination comparatively to the Bayesian rule. Transfers of belief are illustrated by Fig. 5. Each link is numbered and described here after. Initially the cells are assigned with vacuous mass $m_{\Omega}$. When a cell is observed, the belief is transferred to $F$ or $O(1,2)$. If conflicting information is observed, masses are transferred to the empty set $(3,4)$. The normalization process distributes proportionally the conflicting mass on the other subsets $(5,6,7)$. With probabilities, two belief transfer are possible : from $F$ to $O$ (1') or from $O$ to $F\left(2^{\prime}\right)$ during the change of status of the cell. So, ignorance is defined by an equal probability for $F$ and $O$.

These remarks lead us to study the dynamic of the grid using the evidential framework. Three main advantages can be noticed:

- A high value for mass on empty set $(\emptyset)$ highlights a change of state of the cell $(3,4)$. In a dynamic environment, it is a mean of detecting moving objects on the grid.

- Thanks to the conflicting mass redistribution $(5,6,7)$, the fusion rule acts as a filter: the state of the cell doesn't change immediately. This provides robustnes against outliers.

- The unknown state (e.g. a not-observed cell) is managed independently from the distribution of the beliefs between $F$ and $O$ which corresponds explicitly to contradictory information.

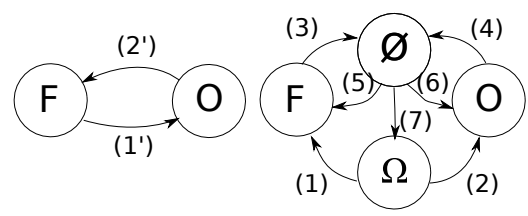

Fig. 5: Transfer of belief during the fusion process. Left: probabilistic framework, right: evidential framework

The approach uses the fact that a moving object occupies different cells along the time The motion of an object is represented by the conflict resulting from the conjunctive fusion, as detailed in section III-A. The empty mass is computed by equation 6 . One can notice two different terms. The first term $C_{1}$ refers to the case of a free cell becoming occupied (link 3 on Fig. 5) and represents the mobile object.
The second term $C_{2}$ corresponds to the case of an occupied cell becoming free (link 4 on fig. 5) and represents past the position of the mobile object.

$$
\begin{aligned}
& m_{\cap M, k}(\emptyset)=C_{1}+C_{2} \\
& C_{1}=m_{S^{\prime}, k}(O) \cdot{ }^{\alpha} m_{M, k-1}(F) \quad F \text { becomes } O \\
& C_{2}=m_{S^{\prime}, k}(F) \cdot{ }^{\alpha} m_{M, k-1}(O) \quad O \text { becomes } F
\end{aligned}
$$

Figure 6 presents a simulation of the evolution of the mass functions for one cell. Top figures display the ground truth ( 1 means that the cell is occupied, 0 means it's free) and a simulated ScanGrid detection. The central plot shows the result of the conjunctive combination, illustrating the conflict generation process and the bottom figure shows the final result of the DS combination. In the first step, the MG cell, initialized with the vacuous mass, converges to a free state according to the SG. Then, since no change occurs, it remains stable. At scan 11 the state changes from $F$ to $O$. Contradictory information creates a part of conflict $C_{1}$ (first term in Eq. 6), the other part of the mass remains on $F$. Thanks to the normalization, the final state of the MG cell doesn't change, whereas the conflict points out an oncoming object. When the SG remains on the same state during such a time, it forces the MG to change from $F$ to $O$ as shown between time 15 and 18 . The following step does the inverse process.

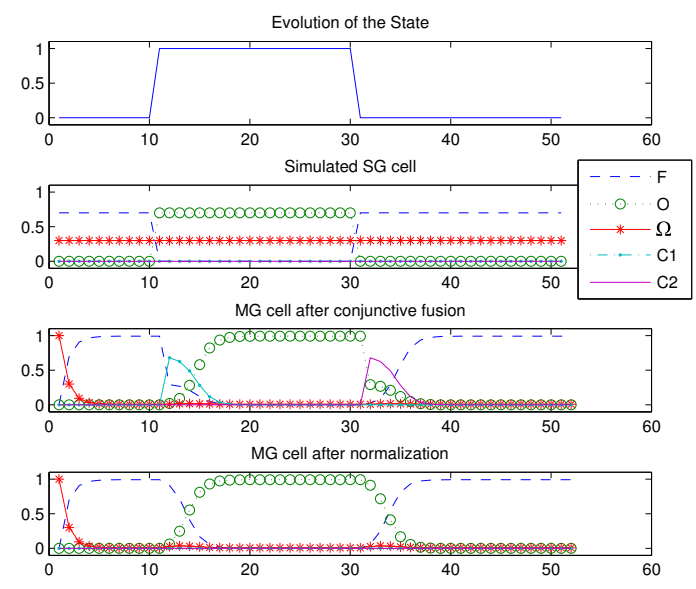

Fig. 6: Simulation of the evolution of the mass function $\left(\lambda_{M D}=\lambda_{F A}=0.7\right)$

Figure 7 shows the result of the detection based on this method. The top figure shows the camera image of the scene, the bottom figure represents the grid containing mobile object (red cells, $C_{1}$ ) and mobile object past positions (blue cells, $C_{2}$ ) in a local frame. The ego vehicle is represented by the green square at the bottom of the grid and is heading up. The detected objects are filtered using a precise road map in order to keep only mobile objects on the road (white cells). One can see that the direction of the travel has been correctly detected thanks to the management of the conflict (from the blue to the red). 


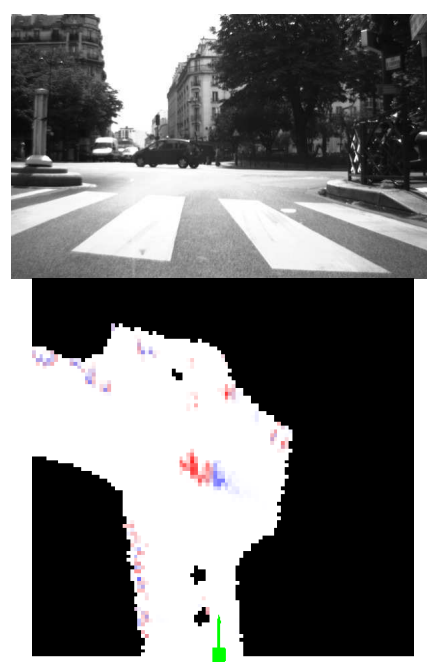

Fig. 7: Result of the detection of a vehicle crossing the street

\section{RESUlTS AND COMPARISON}

The presented algorithm was tested on a set of data acquired with a real vehicle in urban conditions and was compared with an approach that uses accumulation [16]. It is currently implemented in Matlab and runs offline. The data set is a 20-minutes long sequence, acquired in collaboration with the French Geographic Institute (IGN) in Paris. The vehicle follows a reference track repeated 3 times. The results presented were obtained during the first loop.

The data used are provided by three sensors. Two of them are really used by the algorithm: Lidar ALASCA XT and Applanix positioning system. To synchronize the data between the different sensors, time stamps in time GPS have been used. The lidar has been arranged to have its lowest layer horizontally. This means that, if we assume a low pitch, we do not have to consider the floor. The acquisition frequency is $15 \mathrm{~Hz}$ with a maximum angular resolution of $0.25^{\circ}$ in the vehicle front and $1^{\circ}$ on the sides. The Applanix positioning system consists of two GPS, an IMU and an odometry sensor. A camera has been installed just below the lidar located on the front bumper and was triggered by the lidar. So far, the camera is not used for treatment and is just used for validation and visualization of the scene.

The SG covers a distance of 100 meters and the angular field of $180^{\circ}$ with a resolution of $0.5 \mathrm{~m}$ wide and $1^{\circ}$ angle. MG used covers an area of $800 \mathrm{~m} \times 700 \mathrm{~m}$ with a resolution of $0.5 \mathrm{~m} \times 0.5 \mathrm{~m}$. The discounting factor $\alpha$ was set such that the time constant $\tau=1.3 \mathrm{~s}$. The $\lambda_{M D}$ was computed considering that the angle $\varphi=0.25^{\circ}$ and $\beta=0.5^{\circ}$ and the $\lambda_{F A}$ was set to 0.5 .

One sequence of the results is presented through Figures $8 \mathrm{a}, 8 \mathrm{~b}$ and $8 \mathrm{c}$. At each time step, the front-view camera image is displayed and two grids are plotted (Left: the accumulation approach and Right: the evidential approach). Each grid contains the detected mobile cells on a MG replaced in a local frame They represent an area of $50 \mathrm{~m}$ by $50 \mathrm{~m}$ in front of the vehicle, with a resolution of $0.5 \mathrm{~m}$. We use a prior accurate map provided by IGN to extract the drivable space. The white cells denote the cells that belong to road map, the black ones denote the cells which are out of the road. The red cells indicate the presence of a mobile object. Again, the ego vehicle is represented by the green square at the bottom of the grid and is heading up. The detected moving objects are circled in the camera view and a link indicates the cluster of cells that are represented. During the sequence, the ego vehicle is moving at the speed of $35 \mathrm{~km} / \mathrm{h}$ and crosses two cars and one van.

On Figure 8a, both accumulation and evidential algorithm detect correctly the van. The left side of the car is more recognizable with the evidential approach.

Figure $8 \mathrm{~b}$ shows an interesting phenomenon. The car in the background is correctly detected by both methods. The van is properly detected by the evidential method but poses serious problems for the accumulation method. The van is seen by the lidar (the data used are the same) but most cells are classified as static, this is because the van presents a large surface and produces a high number of laser measurements which increment rapidly the accumulation of the cells that exceeds the threshold between mobile and static.

Figure $8 \mathrm{c}$ shows similar results with a better detection in the accumulation approach, but the evidential one remains more comprehensive. We can see the shape of vehicles and also see the scanning effect. Indeed, the front of vehicles form a spike, like a "V". Because the sensor scans clockwise, the left side is scanned before the right side. If the vehicle moves, it produces this effect.

A global comparison of the two methods during the complete sequence exhibits that mobile objects are detected with their global shape when using the evidential approach. Moreover, a nice quality of this approach is that its tuning is easier to do compared to the accumulation strategy that needs the specification of several thresholds. The tuning parameters necessary for the evidential detections are high level with a physical interpretation. These results are promising and this qualitative study shows the added value of the use of evidential grids for moving object detection. Next experimentation with ground truth will be considered in order to give quantitative results on this method.

\section{CONClusion}

This paper has presented a vehicle perception scheme based on both local and global grid interactions able to detect motion in the surrounding of the vehicle by using an evidential framework. The main contribution of this paper is the presentation of a smart way for detection of moving objects by the means of a grid framework. The DS theory provides interesting tools to manage evidentials in occupancy grids which makes the detection simpler and more robust. The determination of the motion direction using the two kinds of conflict generated by the fusion process is the main perspective of this research. We plan also to study different decision strategies able to provide robustness to false alarms. In its current implementation, the main drawback of this method is that it needs a very precise pose, provided in this experimentation by an Applanix system. One perspective 
is so to analyze how a dead-reckoning localization method, using for instance lidar odometry, will degrade the method.

\section{ACKNOWLEDGMENTS}

This work has been supported by ANR (French National Agency) CityVIP project under grant ANR-07_TSFA-01301 . The system has been tested on sequences recorded by the test platform "STEREOPOLIS". The authors would like to thank the CityVIP group.

\section{REFERENCES}

[1] S. Blackman and R. Popoli, Design and Analysis of Modern Tracking Systems. Artech House, 1999.

[2] C. Hoffmann, "Fusing multiple $2 \mathrm{~d}$ visual features for vehicle detection," in Proc. IEEE Intelligent Vehicles Symp, 2006, pp. 406-411.

[3] F. Fayad and V. Cherfaoui, "Tracking objects using a laser scanner in driving situation based on modeling target shape," IEEE Intelligent Vehicles Symposium, 2007.

[4] S. A. Rodriguez Florez, V. Fremont, P. Bonnifait, and V. Cherfaoui, "Visual Confirmation of Mobile Objects Tracked by a Multi-layer Lidar," in IEEE Conference on Intelligent Transportation Systems IEEE Conference on Intelligent Transportation Systems (IEEE ITSC2010), Portugal, 09 2010, p. IEEE ITSC2010.

[5] Y. Bar-Shalom, Multitarget-Multisensor tracking : Applications and Andvances. Artech House, 2000.

[6] J. Leonard, J. How, S. Teller, M. Berger, S. Campbell, G. Fiore, L. Fletcher, E. Frazzoli, A. Huang, S. Karaman, O. Koch, Y. Kuwata, D. Moore, E. Olson, S. Peters, J. Teo, R. Truax, M. Walter, D. Barrett, A. Epstein, K. Maheloni, K. Moyer, T. Jones, R. Buckley, M. Antone, R. Galejs, S. Krishnamurthy, and J. Williams, "A perception driven autonomous urban vehicle," Journal of Field Robotics, vol. 25, no. 10, September 2008.

[7] C. Coue, C. Pradalier, C. Laugier, T. Fraichard, and P. Bessiere, "Bayesian occupancy filteing for multitarget tracking : an automotive application," International Journal of robotics research, vol. 25, no. 1, pp. 19-30, 2006.

[8] A. Elfes, "Using occupancy grids for mobile robot perception and navigation," Computer, vol. 22, no. 6, pp. 46 - 57, 1989.

[9] T. Gindele, S. Brechtel, J. Schröder, and R. Dillmann, "Bayesian occupancy grid filter for dynamic environments using prior map knowledge," Intelligent Vehicles Symposium, 2009 IEEE, 2009.

[10] D. Pagac, E. M. Nebot, and H.Durrant-Whyte, "An evidential approach to map-building for autonomous vehicles," IEEE Transactions on Robotics and Automation, vol. 14, no. 4, pp. 623-629, 1998.

[11] T. Yang and V. Aitken, "Evidential mapping for mobile robots with range sensors," vol. 55, no. 4, pp. 1422-1429, 2006.

[12] P. Smets and R. Kennes, "The transferable belief model," Artificial Intelligence, vol. 66, pp. 191-234, 1994.

[13] O. Bezet and V. Cherfaoui, "Time error correction for laser range scanner data," in 9th International Conference on Information Fusion (FUSION 2006), 2006.

[14] S. Thrun, W. Burgard, and D. Fox, Probabilistic Robotics (Intelligent Robotics and Autonomous Agents), 2001.

[15] J. Moras, V. Cherfaoui, and P. Bonnifait, "Credibilist occupancy grids for vehicle perception in dynamic environments," in IEEE International Conference on Robotics and Automation (ICRA 2011), Shanghai, May 9 to 13 2011, pp. -, to appear.

[16] J. Moras, V. Cherfaoui, and P. Bonnifait, "A lidar Perception Scheme for Intelligent Vehicle Navigation," in 11th International Conference on Control, Automation, Robotics and Vision, Singapour Singapore, 122010

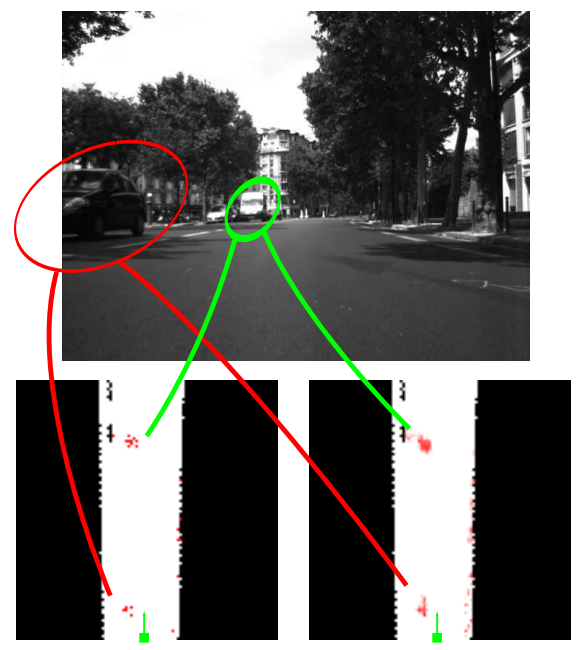

(a) Result of the detection time $\mathrm{k}$

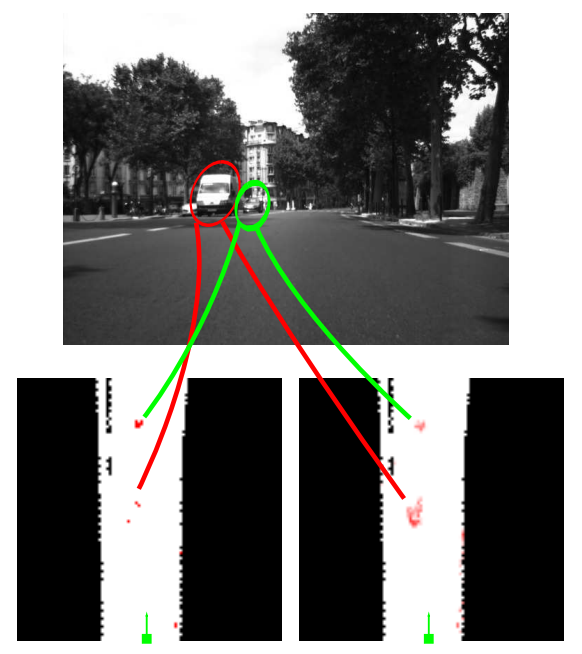

(b) Result of the detection at time $\mathrm{k}+10$

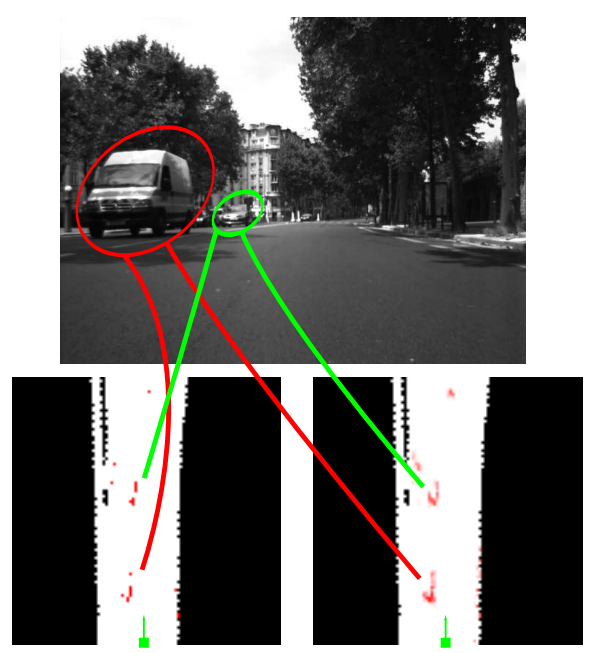

(c) Result of the detection at time $\mathrm{k}+20$

Fig. 8: Result of mobile object detection: top: camera view, left grid: accumulation detection results and right grid: evidential detection results. 